\title{
User-friendly locations of error messages in web forms: Put them on the right side of the erroneous input field ${ }^{\text {th }}$
}

\author{
Mirjam Seckler, Alexandre N. Tuch, Klaus Opwis, Javier A. Bargas-Avila*,1 \\ Center for Cognitive Psychology and Methodology, Department of Psychology, University of Basel, Switzerland
}

\section{A R T I C L E I N F O}

Article history:

Received 4 April 2011

Received in revised form 17 February 2012

Accepted 8 March 2012

Available online 30 March 2012

\section{Keywords:}

Error message location

Online forms

Display errors

Form usability

Form validation

Interaction design

\begin{abstract}
A B S T R A C T
There are many ways of placing error messages in web forms. A study of web conventions shows that the most common approach is to display error messages embedded in the form at the top of the entire form. Six frequent locations (right, left, above and below the erroneous input field, as well as on the top and at the bottom of the form) were tested in an online study with $n=303$ participants. Results of efficiency, effectiveness and satisfaction show that the locations near the erroneous input field lead to a significantly better performance than the error messages on the top and at the bottom of the form; in addition error messages on the right side of the erroneous input field were subjectively evaluated as the most satisfying and intuitive by participants. The results indicate possible improvements for online shops, where error messages are currently mostly placed on the top of the form.
\end{abstract}

(c) 2012 British Informatics Society Limited. Published by Elsevier B.V. All rights reserved.

\section{Introduction}

Today many companies ask people to submit crucial information via web forms. For a successful interaction with customers web forms are therefore an important component of online shops, contact points, social applications and similar applications. There are several types of web forms: (1) Registration forms are often used as gatekeepers to social communities, (2) checkout forms stand between people and companies' products, and (3) data input forms are used to share or search information (Wroblewski, 2008). Although web forms are very common, people usually do not like to fill them out (Wroblewski, 2008). They are seen as obstacles between what people want and how people can get it (e.g. buy a book, apply for a job).

One of the most important factors of a web form are error messages (Wroblewski, 2008). They point at problems and show how to solve them. The main goal is to get the users back to their task as quickly as possible (Jarrett and Gaffney, 2008; Wilska, 2004). Error messages are one of the most frustrating experiences when using computers (Ceaparu et al., 2004; Lazar and Huang, 2003). Although there are several guidelines on how to design a good web form (e.g. Bargas-Avila et al., 2010), error messages usually cannot be avoided completely. Jarrett (2008) distinguishes differ-

\footnotetext{
This paper has been recommended for acceptance by Paul Cairns

* Corresponding author.

E-mail address: javier.bargas@unibas.ch (J.A. Bargas-Avila).

1 This author currently works for Google Inc.
}

ent types of error messages in web forms, for instance typing errors, transcription errors and send errors. Error messages can vary on a broad diversity of features, including the format and the type (text style, size and color), use of graphical features, the location in the form, the wording and the time when an error message appears.

In the last years many aspects of usable web form design have been researched. Topics like cultural adaptability (Recabarren and Nussbaum, 2010), date entry field formatting (Bargas-Avila et al., 2011a; Christian et al., 2007; Couper et al., 2004), error message timing (Bargas-Avila et al., 2007), field format restrictions (Bargas-Avila et al., 2011c), label alignment (Das et al., 2008), multiple option selection (Bargas-Avila et al., 2011b), mandatory field highlighting (Pauwels et al., 2009; Tullis and Pons, 1997), question types (Reja et al., 2003) and response option formats (Healey, 2007; Heerwegh and Loosveldt, 2002) have been subject to empirical evaluation. While there is an increasing number of empirical studies in this field, the topic of where an error message is best placed within a web form was rarely explored. The presented study addresses this topic with an experimental online study.

\section{Theoretical background}

There are many guidelines and a few studies that address the usability of error messages, concerning often the phrasing, the timing, the design and the location of error messages. Brown (1983) was one of the first who recognized the importance of error messages. His studies showed that little forethought is given to the 
production of error messages, as well as to the user' potential recovery after an error has occurred. Twenty years later, Lazar and Huang (2003) analyzed browser error messages. They conclude that the vast majority of the analyzed messages still do not meet the most basic guidelines for a satisfying user experience. Many guidelines stress that an error message must be precise, constructive and polite (Lazar and Huang, 2003; Nielsen, 2001; Linderman and Fried, 2004). This is supported by Wenger (1991), who showed that users who experienced an inconsistent error message expressed intense negative affective responses. Tzeng (2004) showed that if a system apologizes for displaying error messages, this helps to create more desirable psychological experiences for the users. Facing the question, if an error message should appear immediately or after the form submission, the International Organization for Standardization recommends to show the error message immediately after leaving a field (ISO-9241, 1996-2002). These guidelines are contradicted by Bargas-Avila et al. (2007), who showed that the best way of presenting error messages is to provide the erroneous fields after users have completed the entire form. Guidelines concerning visual design attributes of an error message emphasize the importance to attract users' attention and recommend therefore the color red and a bold font for the design of an error message (Crawford et al., 2005; Becker and Mottay, 2001; Padilla, 2005).

Also the location of error messages can have a major impact on the user experience (Biddle, 2007). All the same, at the moment there is no standard way of presenting error messages within web forms. This leads to inconsistencies across different sites and causes confusion (Biddle, 2007).

The different ways of presentation can be grouped into four main approaches:

(1) Embedded error messages at the top or the bottom of the form: An error message appears at the top of the page before the first form field or label (see Fig. 1). Less common, an error message appears at the bottom of the form, after the last form field.
(2) Embedded error messages next to the form field: There are four different ways how an error message can be displayed within the body of the form (see Fig. 2). There is the possibility to locate the error message on the left (Location $A$ ) or the right side (Location $B$ ) of an erroneous input field, if the label stands on the top. Further, there is the option to display the error message above (Location $\mathrm{C}$ ) or below (Location $\mathrm{D}$ ) the erroneous input field. If the label stands above the erroneous input field, the error message is usually located between the label and the erroneous input field.

(3) Pop-ups (alert boxes): Pop-ups consist of new web browser windows that display an error message (Biddle, 2007; Jarrett, 2008). The pop-up window opens in front of the original form and contains the error message and a close button. Before users can continue filling the form, they have to confirm the message.

(4) New page: An error message can be displayed on a new page. Usually theres also a link back to the form.

There are several guidelines concerning the location of error messages, partly contradictory. According to Padilla (2005) a location at the top of a page is commonly recognized as standard and can help to clearly distinguish the error message from the rest of the application's user interface and capture the user's attention. Nielsen (2001), however, claims that users look at the page's actionable part first (i.e. the area with the form fields). Thus, a location at the top of a page is not recommended because users do not notice the error message at this location. Crawford et al. (2005) emphasize that error messages should always be placed on the screen in a location where they are likely to be seen and appropriately attributed to the correct question. Featherstone (2005) suggest that placing the error message to the right of the field supports easy scanning. Wroblewski (2008) recommends a combination of an error message at the top of the form as prominent placement and a second message next to an erroneous input field to additionally highlight this field. Biddle (2007) dissuades from using pop-up windows informing the user which fields need
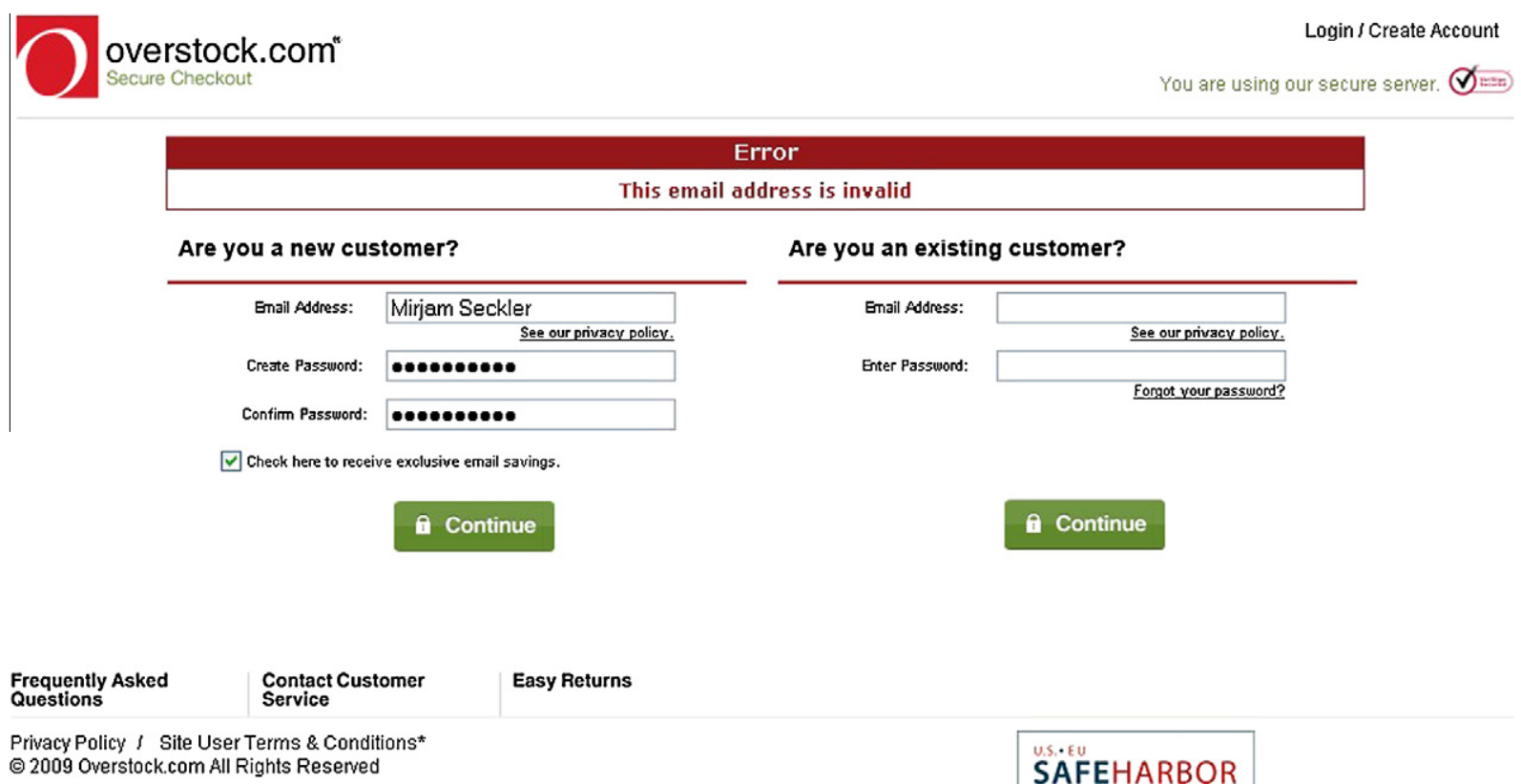

Are you an existing customer?

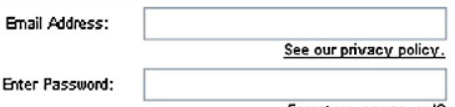

Forgot your password? 


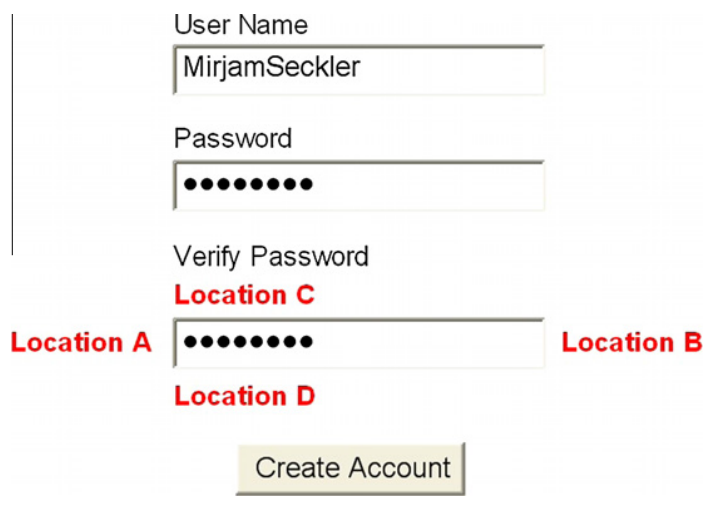

Fig. 2. Four possible locations for error messages next to the erroneous input field (approach 2).

correction. Users tend to close the pop-up windows without reading the message (a phenomenon also reported by Bargas-Avila et al. (2007)). Furthermore, pop-up windows are often used for advertisement.

Other authors propose that different kinds of error messages should have different locations. According to Wilska (2004) popup windows are well-suited for error messages that inform users of problems they cannot fix or that require only basic action. If the problem at hand requires them to do something more substantial, for instance to retype information, Wilska (2004) recommends to use an on-screen error message directly above or next to the field. Jarrett (2008) distinguishes between even more different types of error messages. On the one hand, pop-up windows or top of the page messages are well-suited for sending errors and privacy errors because there is more space for explanations. On the other hand, an error message next to the field is preferable for typing and transcription errors (and for a small number of category errors).

To the authors' knowledge, the only empirical study about the location of error messages is from Mockovak (2005) - an unreviewed tech report. Mochovak used an existing survey web form and compared error messages at the top of a page or directly under an item. The results from 42 participants showed that up to $40 \%$ missed the initial appearance of error messages, but the approaches for presenting the error messages did not result in statistically significant differences. The authors explained this high missing rate as a result of getting familiar with the interface and the general task, as well as a possible change blindness effect when encountering error messages. Change blindness is defined as the failure to detect what should be an obvious visual change in the visual field (Simons and Rensink, 2005) and this effect might also occur when perceiving error messages (Hudson, 2001). There was also no significant difference in the efficiency (total time spent dealing with the error message). Participants preferred having error messages displayed under an item. Mochovak's study provides first insights about where to place error messages. A shortcoming of this study is that in reality there are more than the two locations for error messages. We do not know how these two possibilities perform in comparison with other locations. Furthermore, the study deals mainly with soft error messages that allow users to continue with their task without any need to correct the error. However, there might be a difference between soft and hard error messages in regard to user performance.

The goal of this study is to examine different error message locations. In order to clarify the practical relevance and to allow an accurate formulation of hypotheses, the possible locations of error messages were first evaluated in a study about web conventions, reported in the following section.

\section{Study of web conventions}

To determine which are the most common locations of error messages, we decided to analyze the 100 most popular online shops using Alexa.com (Alexa, 2010) and 100 randomly selected online shops using StumbleUpon.com (StumbleUpon, 2010).

\subsection{World's 100 top shopping web sites}

The Alexa traffic rank website lists the most popular websites of the world. Popularity means a combination of average daily visitors and page views over the past month. There is a category shopping where the 100 most popular shopping websites are listed (e.g. Amazon, Ikea and Overstock). Similar to Roth et al. (2010), we used this list to determine the error message location in each online shop. Because some shops have different top-level domains (TLDs), they are listed twice and more. In this case, only the first top-level domain was used for this study.

\subsection{Hundred shopping websites at random}

To avoid using only popular websites, we added another 100 randomly selected websites using StumbleUpon, a discovery engine that finds and recommends web content to its users. There is a category shopping with an unknown number of indexed sites. For the analysis, only sites from this category were used. We stumbled through the websites by clicking the stumble-button until 100 websites were registered.

\subsection{Procedure}

For each of the 200 shopping sites, we analyzed the placement of the error messages with the following procedure: (1) Access the website, (2) buy a product, (3) click the checkout button and (4) submit the check-out form with wrong and missing information while checking at which location the error messages appear. Then the ordering process was aborted. If a login creation was mandatory to be able to shop, then this login process was used for producing the error messages.

\subsection{Results}

The web conventions show that there are several approaches where to place an error message nowadays. Four single approaches and a combination of the two embedded approaches (see Table 1) can be identified. Seventeen websites from the Alexa group and 18 websites from the StumbleUpon group had to be excluded from the analyses either because of multiple top-level domains, because they were not a web shop (coupon collections or communities),

Table 1

Approaches for the location of error messages in online shops (June, 2010).

\begin{tabular}{lrrl}
\hline Approach & Top sites & Random sites & Total \\
\hline Embedded, outside the body & & & \\
Top of the form & 40 & 35 & $75(45.5 \%)$ \\
Bottom of the form & 1 & 3 & $4(2.4 \%)$ \\
Embedded, next to the erroneous input field & & \\
Left of the erroneous input field & 1 & 0 & $1(0.6 \%)$ \\
Right of the erroneous input field & 5 & 6 & $11(6.7 \%)$ \\
Above erroneous input field & 4 & 0 & $4(2.4 \%)$ \\
Below erroneous input field & 5 & 7 & $12(7.3 \%)$ \\
Embedded, combined & 17 & 2 & $19(11.5 \%)$ \\
Pop-up (alert box) & 10 & 26 & $36(21.8 \%)$ \\
New page & 0 & 3 & $3(1.8 \%)$ \\
Total & 83 & 82 & $165(100 \%)$ \\
\hline
\end{tabular}


because they were a forwarding site or because they were not accessible at the time. Embedded messages represent the most common approach (see Table 1), with the placement at the top of the form being used in almost half of the cases. In total, the error messages embedded in the form cover $64.9 \%$ of all cases. The following placements differ significantly between top sites and random sites: error messages above the input field $\left(\chi^{2}(1, N=165)=4.05, p=.044\right)$, popup error messages $\left(\chi^{2}(1, N=165)=9.35, p=.002\right)$ and combinations of error messages $\left(\chi^{2}(1, N=165)=13.18, p=.001\right)$.

\subsection{Conclusions}

The study of web conventions showed that currently there are six common embedded approaches to present error messages. In addition there are some combined versions (e.g. at the top and below the erroneous field), as well as some cases where pop-up messages or new pages are used.

Due to feasibility reasons it was decided not to test combinations of different locations for the time being. Although pop-up messages are quite frequently found (21.8\%), we refrained from testing this approach. The study from Bargas-Avila et al. (2007) already showed that pop-ups are disadvantageous in many ways. If there is more than one error in a form, then a pop-up has a negative effect, because users have to remember all mistakes. For the same reason it was decided not to test the new page approach.

Therefore, this study compares the six different locations embedded in the form (top, bottom, left, right, above and below).

\section{Main study}

The present study aims to investigate how six embedded error message locations (see Fig. 3) differ regarding efficiency, effectiveness, satisfaction and preference ratings. Therefore an online study with an online shop, an ordering process and a final questionnaire was developed. During the ordering process four inevitable error messages were shown analogous to the study by Mockovak (2005) and Bargas-Avila et al. (2007).

\subsection{Hypotheses}

We used the following hypotheses:

- Efficiency-hypotheses. Supposing that error messages near the erroneous input field (left, right, above and below) quickly direct users' attention to the problem zone (Wilska, 2004) and therefore shorten the search process, these four locations will lead to a significantly shorter time to first click than the other two locations (at the top and the bottom of the form). The fastest interaction should be reached with error messages above and below the erroneous input field, because these locations shorten the scan path and therefore allow rapid processing (see e.g. Penzo, 2006). No significant differences are expected for completion times of the whole form, because the error message location is expected to be a small factor in the entire interaction process (analogous to Bargas-Avila et al., 2007).

- Effectiveness-hypotheses. According to Nielsen (2001), users look at the pages' actionable part first, thus an error message at the top and the bottom of the form are more likely to be overlooked. Therefore it is expected that users will make in this conditions (error messages at the top and bottom) the same error more than once (consecutive errors, see Bargas-Avila et al., 2007). Error messages on the left and on the right of the erroneous input field should be noted more likely because they stand out and therefore the consecutive error rate should be lower for users in these conditions in comparison to the other four.
- Satisfaction-hypotheses. No differences between the six locations are expected for the evaluation of the online shop, again because the error message location is expected not to have sufficient influence to alter the overall evaluation.

- Preference-hypotheses. Error messages at the top and the bottom of the form are expected to have the lowest preference ratings, because they are expected to be inefficient and ineffective.

\subsection{Method}

\subsubsection{Experimental design}

For this study an unrelated samples design was used. The independent variable was the location of the error messages with six levels (right, left, above, below, top, and bottom). The primary dependent variables were user performance (efficiency and effectiveness of corrections), subjective satisfaction with the online shop and subjective preferences of the error message location.

\subsubsection{Measurements}

Efficiency was measured by the time needed to correct an error message (from page load until submit) and the time from load to the first click (using Javascript events) in the field of the error message. Effectiveness was measured by the number of consecutive errors. Finally, subjective satisfaction with the online shop was measured with three validated questionnaires: the WOOS (Yom and Wilhelm, 2004), the NASA-TLX (Hart and Staveland, 1988) and the SAM scale (Lang, 1980). The WOOS questionnaire measures perceived orientation in online shops. It contains seven questions about the structure, efficient location, meaningful naming and orientation in the online shop. Participants rated these seven questions on a 5-point Likert scale. The NASA-TLX is a subjective workload assessment questionnaire consisting of six items asking for the amount of experienced mental, physical and temporal demands, as well as ratings of performance, effort and frustration experienced during task completion (Hart and Staveland, 1988). The questionnaire was implemented without the weighting function to reduce time requirements for participants. Participants rated the six items on visual analog scales which were converted to 100 -point scales. The self-assessment manikin (SAM) is a nonverbal pictorial rating scale with the three dimensions pleasure, arousal and dominance (Lang, 1980) and is the most common tool to measure affect in the field of user experience (Bargas-Avila and Hornbæk, 2011). Subjective preference was measured by presenting all locations with screenshots and asking for the most and least preferred one. For the purpose of a manipulation check, participants were also asked to rate the perceived authenticity of the online shop and to state if they encountered error messages.

\subsubsection{Materials}

4.2.3.1. Online shop. For an authentic online shop experience, a shop for clothing with navigation, product listing pages and shopping basket was programmed (similar to Tuch et al., in press). In total, the shop contained more than 1300 different product items. The screenshot for the start page can be seen in Fig. 4 .

4.2.3.2. Ordering process. The ordering process consisted of five different web forms (welcome, address, dispatch, payment, confirm). The sequence of the forms was taken from Amazon (amazon.com) and shortened (items and wrap were left out). A progress indicator was used to provide users a sense of the scope of the ordering process (Wroblewski, 2008).

The key factor of the experiment were four built-in, unavoidable error messages (see Bargas-Avila et al., 2007; Mockovak, 2005) that appeared all separately on four of the five different forms (see Table 2). Note that on the form "dispatch" no error message was used, to provide a little break to the participants. 


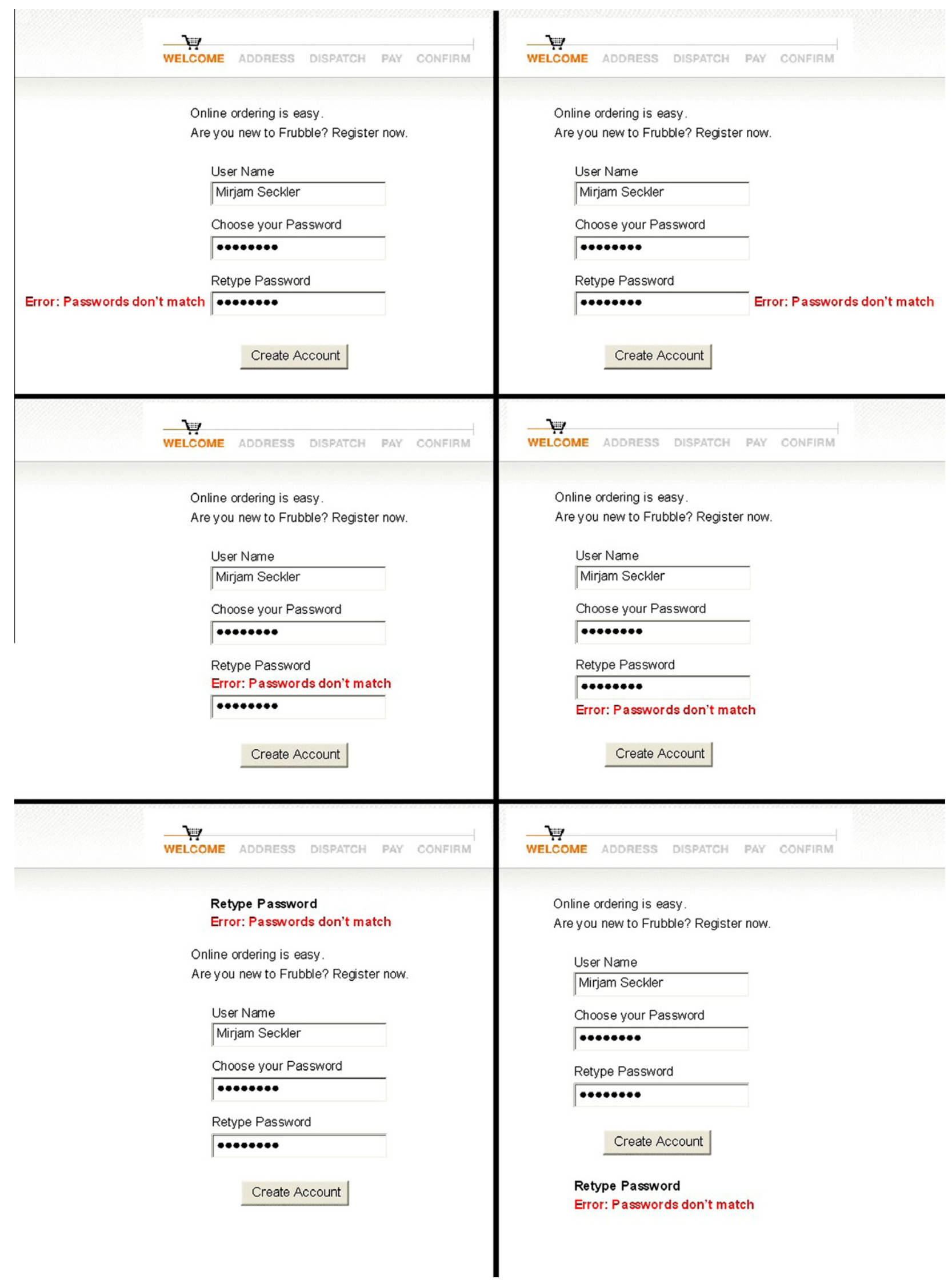

Fig. 3. Example of the six different error message locations (translated by the authors). 


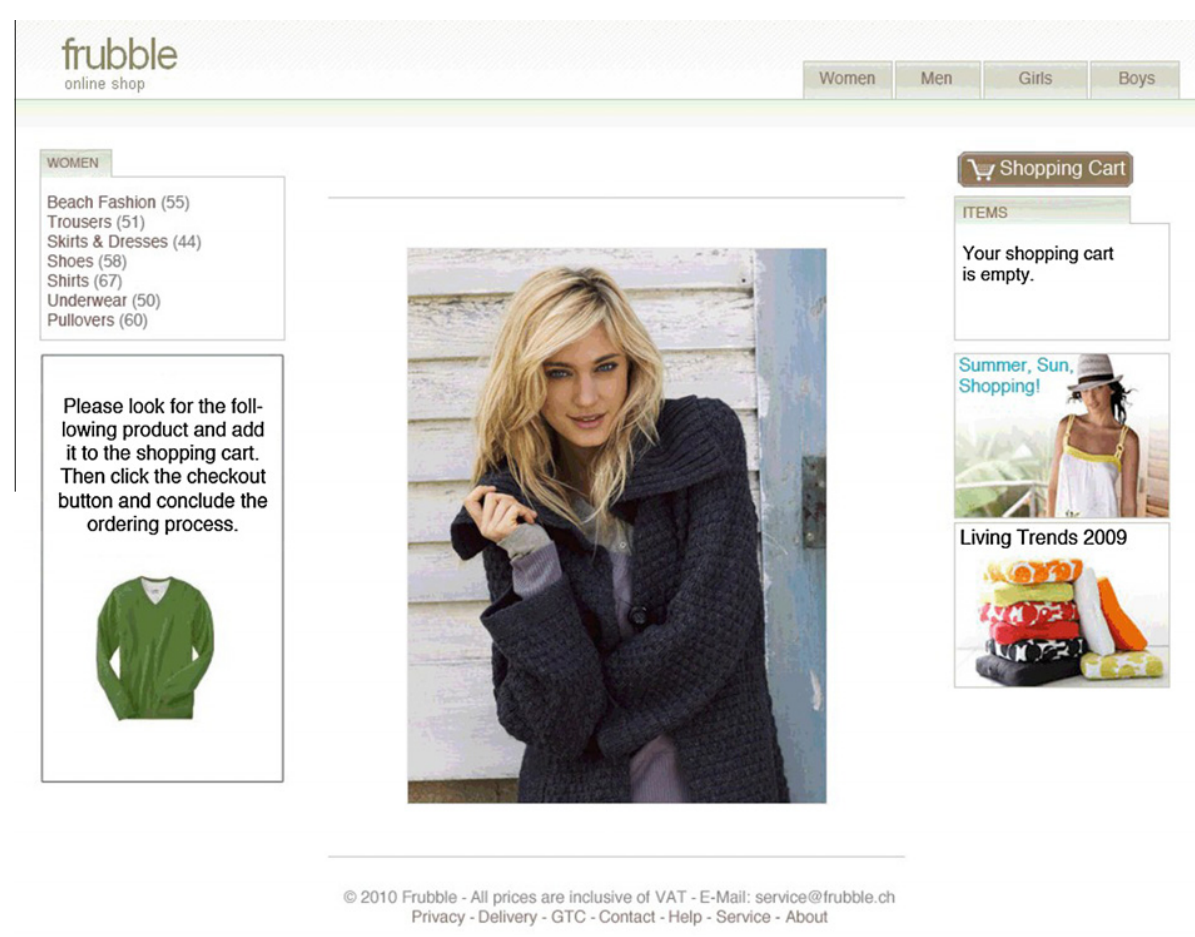

Fig. 4. Start page of the online shop including the task description (translated by the authors).

Table 2

Error messages in the ordering process (translated by the authors).

\begin{tabular}{|c|c|c|c|c|}
\hline $\begin{array}{l}\text { Field (form } \\
\text { name) }\end{array}$ & Error message & Description & Visual stimuli & Type \\
\hline $\begin{array}{l}\text { Password again } \\
\text { (welcome) }\end{array}$ & $\begin{array}{l}\text { Error: passwords do } \\
\text { not match }\end{array}$ & $\begin{array}{l}\text { It was asserted that 'Password' and 'Password again' did not } \\
\text { match }\end{array}$ & $\begin{array}{l}\text { Input fields: 'Password' and } \\
\text { 'Password again' fields empty }\end{array}$ & Typing error \\
\hline $\begin{array}{l}\text { Date of birth } \\
\quad \text { (address) }\end{array}$ & $\begin{array}{l}\text { please use following } \\
\text { format: } 24 / 05 / 2010\end{array}$ & $\begin{array}{l}\text { Day-month-year had to be separated by '/' and day/month with } \\
\text { 2-digits, the year with } 4 \text {-digits (e.g. } 21 / 02 / 1979 \text { ) }\end{array}$ & Input field: 'date of birth' field empty & $\begin{array}{l}\text { System } \\
\text { restriction } \\
\text { error }\end{array}$ \\
\hline $\begin{array}{l}\text { Payment } \\
\text { method } \\
\text { (pay) }\end{array}$ & $\begin{array}{l}\text { is currently not } \\
\text { available }\end{array}$ & $\begin{array}{l}\text { It was asserted that the selected payment method was currently } \\
\text { unavailable }\end{array}$ & Drop down menu: no visual change & $\begin{array}{l}\text { Category } \\
\text { error }\end{array}$ \\
\hline $\begin{array}{l}\text { Confirmation } \\
\text { code } \\
\text { (confirm) }\end{array}$ & $\begin{array}{l}\text { Error: confirmation } \\
\text { code is incorrect }\end{array}$ & It was asserted that the confirmation code (Captcha) was wrong & $\begin{array}{l}\text { Input field: confirmation code empty, } \\
\text { new Captcha was generated }\end{array}$ & $\begin{array}{l}\text { Transcription } \\
\text { error }\end{array}$ \\
\hline
\end{tabular}

Different error message types were used to simulate different problems and to provide a realistic scenario (see Table 2 for different types and exact error messages). Two of the four error messages ("date of birth" and "payment method") demanded an exact reading of the text and were therefore suited to measure effectiveness, because not reading these messages leads to consecutive errors. The other two messages only asked for repeated input.

The error messages were written in red. ${ }^{2}$ The distance between an error message and the erroneous input field was 20 pixels for the left and right location; a line break above the erroneous input field respectively below the field label for the above variant, and a line break below the erroneous input field for the below version. The error messages at the top of the form stood a line break above the form title and were written on two lines. The first line was black and contained the erroneous label; the second line was red and contained the error message. The error messages at the bottom of the form used the same design; the location was a line break be-

\footnotetext{
${ }^{2}$ For interpretation of color in Figs. 1-4 and 6, the reader is referred to the web version of this article.
}

low the next-button. The correct locations were implemented for the following browsers: Internet Explorer 7, Internet Explorer 8, Firefox 3.5, Firefox 3.6 and Safari 4, therefore only users using these browsers were allowed to participate.

\subsubsection{Procedure}

The online experiment took place from October 2010 to November 2010 and was conducted in German. Starting from an introduction page, participants were randomly assigned to one of the six experimental conditions (see Fig. 5) and directed to the online shop. The task in the shop was written on a banner and involved locating one product. The shopping task served only to simulate real shopping experience, though.

After putting the product in the shopping cart, participants could click on a checkout-button. This led them to the checkout process, where each participant was assigned to one of the six experimental conditions (location of the error message). That is, each participant encountered four error messages (see Table 2), and saw these error messages always with the same placement (right, left, above, below, top or bottom). The error messages 


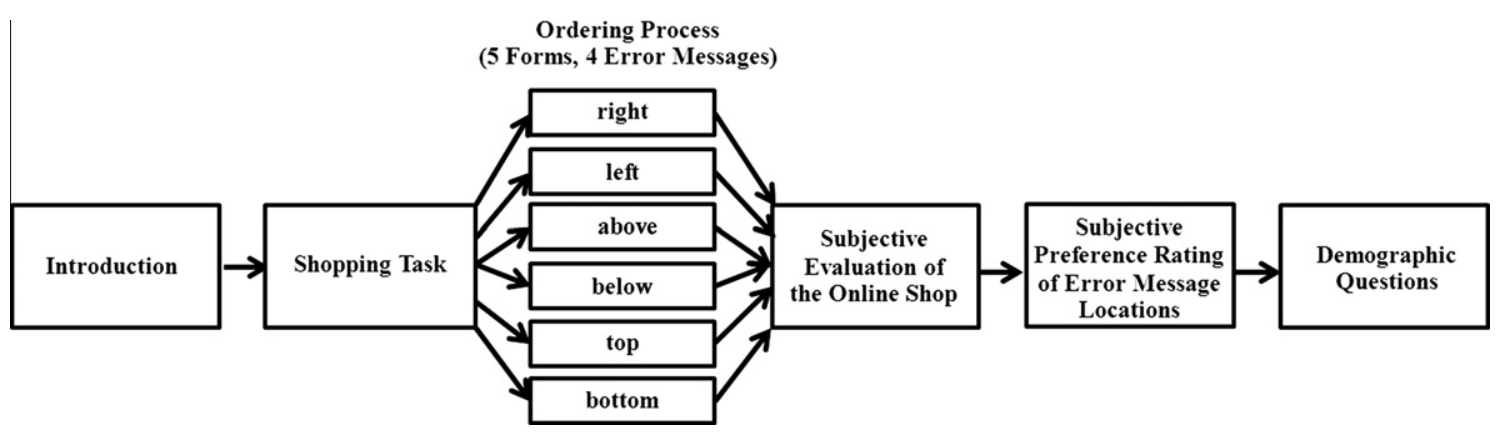

Fig. 5. Overview of the experimental procedure.

appeared after a form was submitted and it was not possible to skip the error messages without correction. After having finished the process, users were directed to the final questionnaire.

First, three ad hoc items about usability ("Please rate how usable the online shop is"), frustration ("Please rate how frustrated you have been while interacting with the online shop") and handling of the online shop ("Please rate how easy or difficult the online shop was to handle") were asked (7-point Likert scales), followed by the WOOS, NASA-TLX and SAM questionnaires. Then, participants had to rate the authenticity of the online shop and were asked if they had noticed the error messages. Furthermore, after the experiment, they were asked for the most favored and the most annoying location of error messages by comparing screenshots of all six locations. At the end, there were some demographic questions.

\subsubsection{Participants}

The participants were recruited from an internal recruiting database, containing the data of people interested in attending studies. An iPhone 4 was raffled between all participants as incentive. The participants were contacted via e-mail containing the participation link. In total 487 people started the experiment, of which 124 aborted the study after the introduction page. Nineteen participants quit after the first error message appeared, 24 later during the ordering process. The error message location did not influence the dropout rate. There was no difference between the experimental conditions, $\chi^{2}(5, N=43)=4.30, p=.507$. Six participants dropped out during the final questionnaire and six participants used a mobile device and were therefore excluded. Another five participants indicated visual color impairment and were also excluded. In total this leads to a dropout rate of $37.14 \%$.

A total of $n=303$ participants were included in the analysis (34\% male, 65\% female, $1 \%$ did not indicate their gender). The mean age was 28 years $(S D=9.99$; range: $15-64)$. The average self-rated computer knowledge on a scale from 1 to $7(1=$ no experience; 7 = expert $)$ was $5.49(S D=0.92)$. Ninety-eight percent of all participants were familiar with the Internet using it several times a week (7\%) or daily (91\%). The average self-rated online shopping knowledge on a scale from 1 to 7 ( $1=$ no experience; 7 = expert $)$ was 4.68
$(S D=1.35)$. Eighty percent of all participants already bought goods in online shops more than five times, $67 \%$ even more than 10 times.

\subsection{Results}

For all statistical tests an alpha level of .05 was used. Furthermore, all data were checked if they met the required conditions for the statistical tests. All time values had to be log-transformed to achieve normal distribution. Differing sample sizes within the statistical values are due to individual missing data values. Before the main analysis, a manipulation check and an analysis of covariates were conducted.

\subsubsection{Manipulation check}

First, the online shop was checked for authenticity. An analysis of variance (ANOVA) for independent samples with the factors error message location and authenticity of the online shop showed no significant differences $\left(F(5,297)=2.18, p=.056, \eta_{p}^{2}=.04\right)$, therefore the manipulation was successful. The average rated authenticity on a scale from 1 to $7(1=$ not realistic; $7=$ very realistic) was $5.12(S D=1.61)$.

Second, a chi-square test with the factors error message location as independent variable and participants indication if they noticed error messages as dependent variable was conducted. The analysis showed that the experimental factor error message location did not lead to different ratings and was therefore successfully manipulated, $\chi^{2}(10, N=302)=11.33, p=.332$.

\subsubsection{Covariates}

The analysis of demographic factors with one-way ANOVAs showed no significant differences between the experimental groups age distribution, computer knowledge, Internet usage and online shopping knowledge. A chi-square test indicated that there are also no significant differences regarding gender distribution over the six conditions, as well as regarding the used browsers.

\subsubsection{Efficiency}

First, an ANOVA for independent samples with the factors error message location as independent variable and total time per form as dependent variable was conducted. All values are shown in Table

Table 3

Average time from load to submit in seconds for each location.

\begin{tabular}{|c|c|c|c|c|c|c|}
\hline Error message & Right & Left & Above & Below & Top & Bottom \\
\hline \multirow[t]{2}{*}{$n$} & $50-51$ & $45-47$ & 51 & $49-50$ & 52 & $51-52$ \\
\hline & $M(S D)$ & $M(S D)$ & $M(S D)$ & $M(S D)$ & $M(S D)$ & $M(S D)$ \\
\hline Password & $18.29(17.41)$ & $18.18(12.57)$ & $15.86(8.33)$ & $14.11(6.15)$ & 17.07 (12.97) & $22.83(21.63)$ \\
\hline Date of birth & $15.28(8.63)$ & $15.67(10.61)$ & $14.53(6.62)$ & $16.16(11.48)$ & $16.41(10.26)$ & $16.73(11.44)$ \\
\hline Payment method & $7.41(4.36)$ & $7.87(6.40)$ & $6.50(3.02)$ & $6.90(4.04)$ & $7.65(3.06)$ & $11.02(9.13)$ \\
\hline Confirmation code & $10.93(4.92)$ & $10.51(4.75)$ & $12.16(10.30)$ & $11.94(6.25)$ & $10.49(4.72)$ & $11.97(5.72)$ \\
\hline
\end{tabular}

Note: Reported values are not log-transformed; statistical tests are based on log-transformed data. 


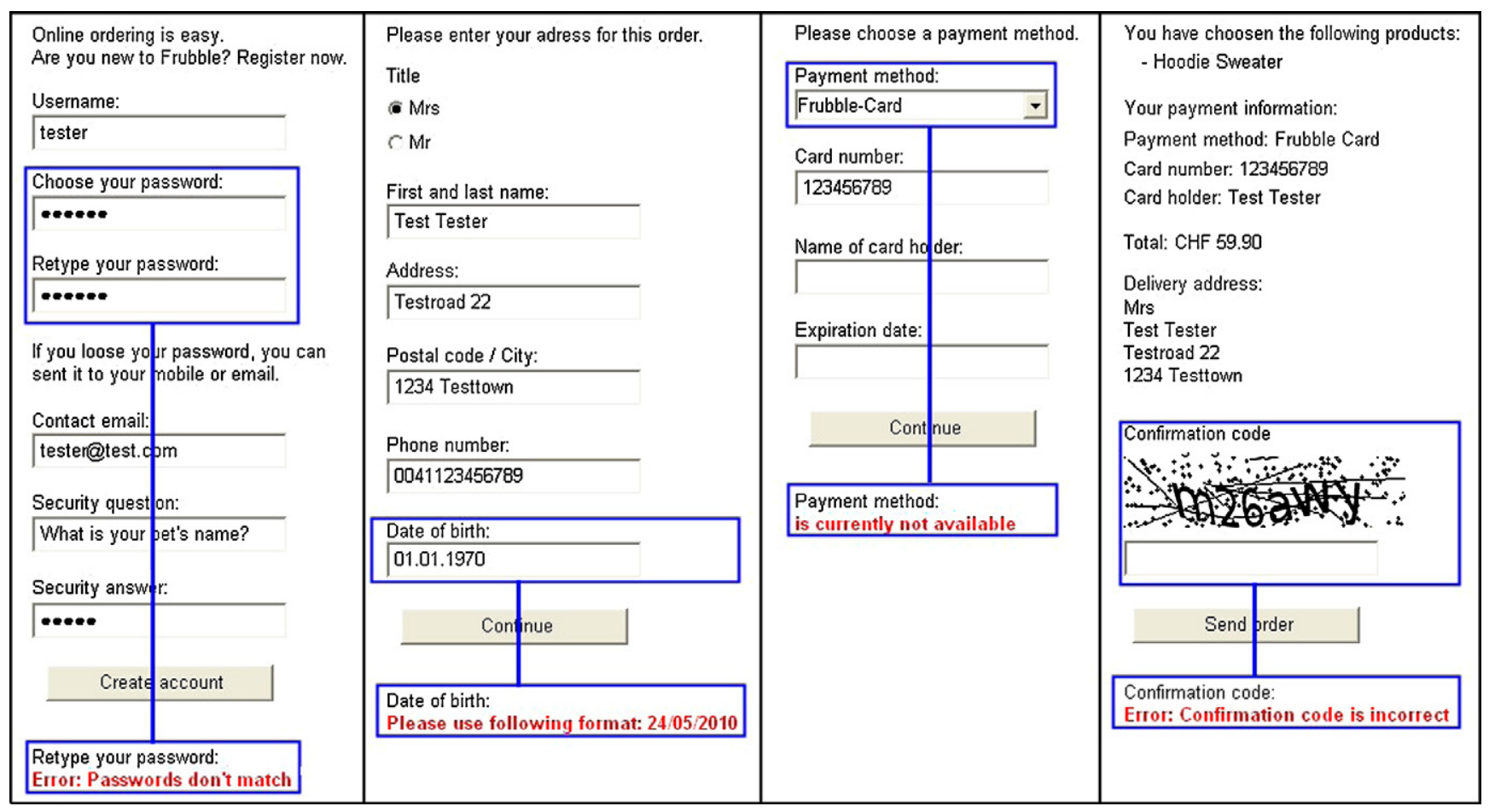

Fig. 6. Distances between erroneous field and error message for the location at the bottom for all four forms.

Table 4

Distances between erroneous field and error message for the location at the bottom.

\begin{tabular}{lllll}
\hline Error message & Distance & $F$ & $p^{* *}$ & $\eta_{p}^{2}$ \\
\hline Password & 375 & $(5,297)=2.42$ & $.036^{* * *}$ & .04 \\
Date of birth & 130 & $(5,295)=.21$ & .959 & .00 \\
Payment method & 305 & $(5,295)=4.95$ & $.001^{* * *}$ & .08 \\
Confirmation code & 150 & $(5,288)=1.58$ & .166 & .03 \\
\hline
\end{tabular}

* In pixels.

** Significant differences in completion time in comparison to the other five locations.

*** $p<.05$.

3. As expected, there were no significant differences for the forms "date of birth" $\left(F(5,295)=.21, p=.959, \eta_{p}^{2}=.00\right)$ and "confirmation code" $\left(F(5,288)=1.58, p=.166, \eta_{p}^{2}=.03\right)$. Unexpected significant results were found for "password" $(F(5,297)=2.42, p=.036$, $\left.\eta_{p}^{2}=.04\right)$ and "payment method" $(F(5,295)=4.95, \quad p<.001$, $\left.\eta_{p}^{2}=.08\right)$. Descriptive data show that for both error messages the location at the bottom led to lower efficiency than the other locations. Post-hoc tests with Scheffé revealed no further significant differences for the password error message; significant differences were found for the payment method error message, indicating that the location at the bottom $(M=11.02,95 \% \mathrm{CI}[9.52,12.52])$ led to a significantly lower efficiency than the error messages above $(M=6.50,95 \% \mathrm{CI}[5.00,7.99], p=.004)$, below $(M=6.90,95 \% \mathrm{CI}$ $[5.39,8.41], p=.014)$ and on the right side $(M=7.41,95 \% \mathrm{CI}$ [5.91,8.91], $p=.049$ ) of the erroneous input field.

Due to the unexpected effect of error message location on total completion time of the form, an additional analysis of the web form design was conducted. The distances between the error message at the bottom and the erroneous field were calculated for all four web forms. The design of each web form with the distances between the erroneous input field and the error message at the bottom is shown in Fig. 6. As Table 4 shows, web forms that led to significant increase in time for the error message at the bottom have a larger distance between the error message and the erroneous input field. These data reveal an obvious insight: The distance between the erroneous field and the error message influences the efficiency of error correction.

Second, the time from loading to the first click in the field that needed to be corrected was analyzed. The ANOVA revealed that there are significant time differences for all forms, "password" with $F(5,220)=7.34, p<.001, \eta_{p}^{2}=.14$, "date of birth" with $F(5,258)=$ 9.47, $p<.001, \eta_{p}^{2}=.16$, "payment method" with $F(5,262)=10.68$, $p<.001, \eta_{p}^{2}=.17$ and "confirmation code" with $F(1,286)=2.78$, $p=.018, \eta_{p}^{2}=.05$. For the descriptive data see Table 5 . A contrast analysis was conducted to test if placing the error messages directly near the erroneous input field resulted in a shorter timespan to the first click than the other two locations (bottom and top). As expected, the error messages at the top and the bottom performed worse than the other locations, password with $F(1,221)=15.98$, $p<.001$, date of birth with $F(1,263)=39.09, p<.001$, payment method with $F(1,263)=41.38, p<.001$ and confirmation code with $F(1,286)=10.14, p=.002$. There was no significant difference between the four locations near the erroneous input field.

\subsubsection{Effectiveness}

According to the study design, only the error messages for the date of birth and for the payment method are relevant for the effectiveness, because only these error messages demanded an exact reading of the text. This is confirmed: There were no significant differences in consecutive error rates between the error message locations for the password $\left(\chi^{2}(5, N=287)=2.08, p=.838\right)$ and confirmation code $\left(\chi^{2}(5, N=303)=3.54, p=.617\right)$ error message. Therefore, these two error messages were disregarded for the effectiveness analysis.

The location of error messages had a significant impact for the correction of an erroneous input field (see Table 6). The results of chi-square tests indicated that there are significant differences between the locations, $\chi^{2}(5, N=271)=11.74, p=.039$ (for "date of birth") and $\chi^{2}(5, N=303)=12.60, p=.027$ (for "payment method"). 
Table 5

Average time from load to first click in seconds for each location.

\begin{tabular}{|c|c|c|c|c|c|c|}
\hline $\begin{array}{l}\text { Error message } \\
n\end{array}$ & $\begin{array}{l}\text { Right } \\
41-47 \\
M(S D)\end{array}$ & $\begin{array}{l}\text { Left } \\
37-43 \\
M(S D)\end{array}$ & $\begin{array}{l}\text { Above } \\
40-51 \\
M(S D)\end{array}$ & $\begin{array}{l}\text { Below } \\
36-46 \\
M(S D)\end{array}$ & $\begin{array}{l}\text { Top } \\
38-52 \\
M(S D)\end{array}$ & $\begin{array}{l}\text { Bottom } \\
34-52 \\
M(S D)\end{array}$ \\
\hline Password & $3.86(2.56)$ & $4.27(3.42)$ & $3.70(2.88)$ & $3.82(1.65)$ & $4.12(2.28)$ & $6.51(2.97)$ \\
\hline Date of birth & $3.24(2.27)$ & $3.04(1.79)$ & $3.33(3.05)$ & $3.57(2.12)$ & 4.96 (3.07) & $4.97(4.29)$ \\
\hline Payment method & $2.89(1.62)$ & $3.05(2.50)$ & $3.01(1.72)$ & $2.77(1.10)$ & $3.87(1.83)$ & $5.33(4.64)$ \\
\hline Confirmation code & $2.90(1.52)$ & $2.40(1.10)$ & $2.91(1.87)$ & $3.00(1.93)$ & $3.41(2.21)$ & $3.56(2.19)$ \\
\hline
\end{tabular}

Note: Reported values are not log-transformed; statistical tests are based on log-transformed data.

Table 6

Error correction successrates.

\begin{tabular}{|c|c|c|c|c|c|c|c|}
\hline Error message & Error correction & Right & Left & Above & Below & Top & Bottom \\
\hline \multicolumn{8}{|l|}{ Date of birth } \\
\hline & Valid (in \%) & 90.7 & 86.7 & 91.3 & 80.0 & 81.8 & 68.8 \\
\hline & Invalid (in \%) & 9.3 & 13.3 & 8.7 & 20.0 & 18.2 & $31.3^{*}$ \\
\hline \multicolumn{8}{|c|}{ Payment method } \\
\hline & Valid (in \%) & 94.1 & 95.7 & 90.2 & 94.0 & 78.8 & 82.7 \\
\hline & Invalid (in \%) & 5.9 & 4.3 & 9.8 & 6.0 & $21.2^{*}$ & 17.3 \\
\hline
\end{tabular}

* $p<.05$.

For further analyses configural frequency analyses with Eye (Grüner, 2008) were conducted. A significant difference between expected and effective frequency was found for the location at the bottom for "date of birth" $(z=2.26, p=.012)$ and for the location at the top for "payment method" $(z=2.05, p=.020)$, indicating that the consecutive error rate for these two locations were significantly higher than for the locations near the erroneous input field.

\subsubsection{Subjective satisfaction}

To test whether the locations differ regarding subjective satisfaction with the online shop, one-way ANOVAs for independent samples were performed. Results indicate that there are no significant differences for the single items usability $(F(5,297)=.94$, $\left.p=.457, \eta_{p}^{2}=.02\right)$, frustration $\left(F(5,297)=1.07, p=.377, \eta_{p}^{2}=.02\right)$ and handling $\left(F(5,297)=1.51, \mathrm{p}=.186, \eta_{p}^{2}=.03\right)$. Likewise, no significant differences were found for the WOOS questionnaire $\left(F(5,297)=1.03, p=.400, \eta_{p}^{2}=.02\right)$ and for the SAM (valence: $F(5,265)=.91, p=.477, \eta_{p}^{2}=.02$; arousal: $F(5,264)=.50, p=.777$, $\eta_{p}^{2}=.01$; dominance: $\left.F(5,264)=.33, p=.896, \eta_{p}^{2}=.01\right)$. However, a difference was found for the NASA-TLX $(F(5,278)=2.49$, $\left.p=.032, \eta_{p}^{2}=.04\right)$. Descriptive data show that the location at the bottom and above the erroneous input field led to higher cognitive load (see Fig. 7). Post-hoc test with Scheff revealed no further significant differences.

\subsubsection{Subjective preference}

Subjective preference data were analyzed with a chi-square goodness of fit test. There were significant differences in the preferred location, $\chi^{2}(5, N=303)=242.84, p<.001$, as well as regarding the most annoying location $\chi^{2}(5, N=303)=130.05, p<.001$. Furthermore, there was a significant difference regarding where participants expect error messages, $\chi^{2}(5, N=303)=103.00$, $p<.001$. All values are shown in Table 7. The least preferred and at the same time the most annoying location was the one at the top, followed by the location at the bottom and on the left. The preferred error message location is on the right side of the erroneous input field, this was also the location where participants indicated to expect the error messages.

Furthermore, we analyzed whether there is an impact of the experimental condition on post-preference judgments. Descriptive data show that there was a tendency to prefer the location of the error message, which the participant has experienced in the as-

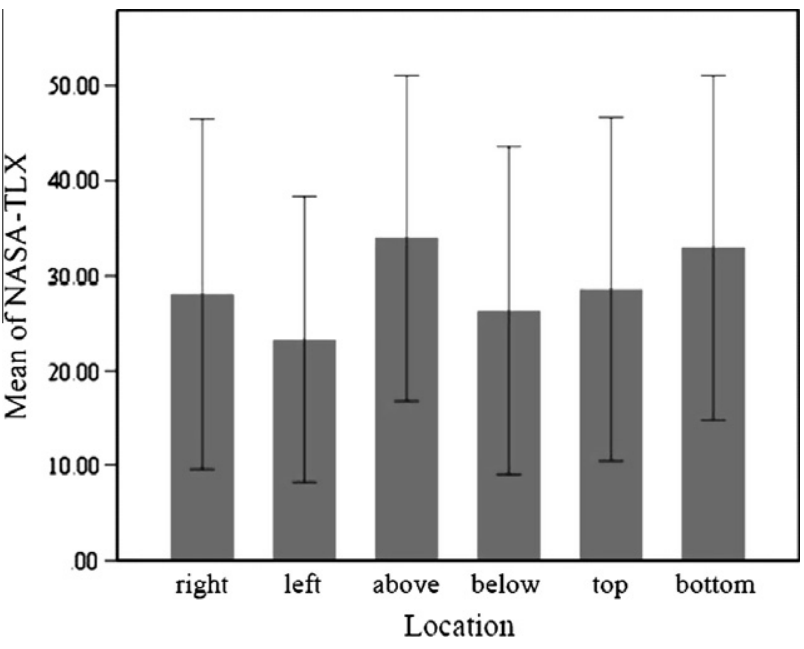

Fig. 7. Mean and standard errors of the Nasa-TLX for each condition.

Table 7

Answers for subjective preference ratings (numbers represent participant count).

\begin{tabular}{lclllrl}
\hline Error message location & Right & Left & Above & Below & Top & Bottom \\
\hline Preferred location & 139 & 27 & 48 & 71 & 7 & 11 \\
Most annoying location & 17 & 83 & 33 & 11 & 100 & 59 \\
Expected location & 104 & 29 & 51 & 70 & 33 & 16 \\
\hline
\end{tabular}

signed experimental condition (see tables in Appendix A for detailed data). Nevertheless, this tendency is not strong enough to dilute the overall statistical effect on location preference. The tendency only exists if compared across the experimental conditions but not if compared within the experimental conditions. However, this tendency of preferring the location of the own experimental condition does not interfere with the observed preference effect. Because the experimental conditions were well balanced, the observed tendency would rather reduce than boost the overall statistical effect. Hence, a within-subjects design, where participants directly experience and compare all the different error messages locations, would most likely lead to even stronger preference effects. 


\section{Discussion}

The study on web conventions showed that there are currently many locations where error messages are displayed in a web form. The main experiment indicates that performance was best when the error message was close to the erroneous input field. In addition error messages on the right side of the erroneous input field were subjectively evaluated as the most satisfying and intuitive by participants, followed by error messages below an erroneous input field.

Regarding the efficiency of the different locations, the error message near the erroneous input field performed significantly better than the other two locations which were further away from the erroneous input field. The results are consistent with the experimental hypothesis and the guideline from Featherstone (2005) but contradict the findings from Mockovak (2005), who did not find a difference between the error message at the top and below the erroneous input field. In our study, the bottom location had even an influence on the overall time used to correct the forms. A closer look shows that this was the case when the erroneous input field was located almost at the top ("password" and "payment method"), leading to a longer distance between the input field and error message.

The analyses of effectiveness confirm these results. The error messages on the top and at the bottom cause higher consecutive error rates. The findings support Nielsen's opinion that users look at the page's actionable part first (Nielsen, 2001) and contradicts Padilla (2005) who claims that error messages on the top of the page capture the user's attention. The results are also comparable with the consecutive error rate in the study from Mockovak (2005), suggesting that not only soft error but also hard error messages can cause high consecutive error rates. Already Hudson (2001) pointed out that error messages are likely to get overlooked and referred to the change blindness effect, which may occur here because the original form is redisplayed with only little changes. The results of the actual study support Hudson's observation as well as Simon and Rensink's statement that objects in a scene that preferentially receive attention, are more likely to be encoded and compared (Simons and Rensink, 2005). It is likely that the error messages near the erroneous input field get more attention and therefore the change blindness effect as well as the consecutive error rates are lower.

As expected, no significant differences were found for the subjective evaluation of the online shop (WOOS, SAM, single items). An evident explanation is that the interaction time with the online shop was too long for an unpopular error message location to have an impact on the entire evaluation. Unexpectedly, the NASA-TLX showed a significant difference between the locations, indicating that the error messages at the bottom and above the erroneous input field caused the highest cognitive load. The former already showed disadvantages in the efficiency and effectiveness and may therefore also lead to high cognitive load. The error message above the erroneous input field may lead to high cognitive load because this location is also used for the field label and can therefore elicit confusion. These findings underline additionally the clear advantage of the locations on the right, the left and below an erroneous input field.

The subjective preference ratings shed more light on the question which location of the three remaining is the best. Subjects clearly preferred and expected error messages on the right side. An explanation can be that because the western reading system goes from left to right, the reaction to an input should be on the right side as well. The second most preferred location is the location below an erroneous input field. This result supports the findings from Mockovak (2005). The disadvantage of this location is the increased vertical space that is needed for displaying a form. The analysis of the subjective preference ratings showed that there is an impact of the experimental condition on post-preference judgments. However, because the experimental conditions were carefully balanced, this tendency should not interfere with the overall subjective preference effect.

Although in this study different types of error messages were used (typing error, system restriction error, category error and transcription error), there was no significant difference regarding usability measures. This contradicts Jarrett's suggestion that different types of error messages fit to different locations (Jarrett, 2008). Although there is more space on the top of a form for an error message, in the actual study this location led to lower efficiency and effectiveness in contrast to the locations near the erroneous input field. Moreover, the suggestion that different locations should be used for different types of error messages may result in inconsistent solutions and may lower predictability.

The results obtained in this study are surprising in the light of the web conventions study. In most online shops, the error messages appear at the top of the form. This may be the case because this location is more easily to program than error messages near an erroneous input field (the exact location of the erroneous input field can be ignored). Yet, the actual study showed problems with the location at the top of the form.

\subsection{Limitations}

There are several limitations of this study that have to be emphasized. First, this study focused only at the location of a text error message. There are miscellaneous graphical possibilities how to point out an error, for instance to frame or highlight an erroneous input field with additional color or symbols. These graphical possibilities may act as endorsement and may help to improve the interaction. Second, due to the fact that we have conducted an online study, we do not know the sizes of the screens on which the participants completed the study. Hence, we are not able to indicate which input fields were above and which were below the fold. Maybe shorter or longer forms may lead to different results. Furthermore, most participants were quite experienced web users. Novice users may have different expectations or show different behavior when handling web forms. Another important factor is that most participants in this study were from Switzerland and therefore the results of the study may not be applied to other cultures. Taking into account that the lettering or the color may have an influence on the perception of error messages, findings may vary in other cultures.

\subsection{Further work}

Further work should explore if the findings from this study can be replicated with longer forms or more than one error message per form. It also may be worth to evaluate a combination of different error message locations. Additionally, eye tracking data would give extended insights where users look and when users notice error messages. Moreover, it would be interesting to investigate the effect of different design options (colors, use of graphical symbols) on the perception of error messages.

\subsection{Conclusion}

In this study important insights were achieved, showing that error messages near the erroneous input field lead to the best performance. Among these error messages, the error message on the right side was evaluated as the most satisfying and expected. The practical implications of the current findings are clear. Comparing 
these results to the findings of the web conventions study, error messages are currently often implemented in suboptimal ways. Only few online shops display the error message on the right side of the erroneous field (6.7\% overall). In most instances the error messages do not even appear directly near the erroneous input field ( $71.5 \%$ overall), but on the top of the entire form ( $45.5 \%$ overall). In the best case this leads to lower ordering speed and customer satisfaction, in the worst case users are not able to complete the ordering process. Many online shops, small shops as well as the world's leading shops, could therefore benefit from an improvement in the placement of error messages.

\section{Acknowledgements}

The authors thank Lars Frasseck for the programming of the online shop and all participants for their valuable contribution to this study.

\section{Appendix A}

See Tables 8-10.

Table 8

Answers for the preferred location in comparison to the experimental conditions.

\begin{tabular}{|c|c|c|c|c|c|c|c|}
\hline & \multicolumn{6}{|c|}{ Preferred location } & \multirow[t]{2}{*}{ Total } \\
\hline & Right & Left & Above & Below & Top & Bottom & \\
\hline \multicolumn{8}{|c|}{ Experimental conditions } \\
\hline Right & 41 & 4 & 2 & 3 & 0 & 1 & 51 \\
\hline Left & 16 & 15 & 11 & 8 & 1 & 1 & 47 \\
\hline Above & 12 & 2 & 15 & 11 & 0 & 1 & 51 \\
\hline Below & 20 & 2 & 7 & 23 & 2 & 1 & 50 \\
\hline Top & 28 & 1 & 11 & 9 & 2 & 1 & 52 \\
\hline Bottom & 22 & 3 & 2 & 17 & 2 & 6 & 52 \\
\hline Total & 139 & 27 & 48 & 71 & 7 & 11 & 303 \\
\hline
\end{tabular}

Table 9

Answers for the most annoying location in comparison to the experimental conditions.

\begin{tabular}{|c|c|c|c|c|c|c|c|}
\hline & \multicolumn{6}{|c|}{ Most annoying location } & \multirow[t]{2}{*}{ Total } \\
\hline & Right & Left & Above & Below & Top & Bottom & \\
\hline \multicolumn{8}{|c|}{ Experimental conditions } \\
\hline Right & 2 & 11 & 7 & 2 & 20 & 9 & 51 \\
\hline Left & 2 & 4 & 8 & 2 & 9 & 14 & 47 \\
\hline Above & 6 & 13 & 1 & 1 & 20 & 7 & 51 \\
\hline Below & 4 & 19 & 6 & 0 & 21 & 11 & 50 \\
\hline Top & 2 & 19 & 4 & 5 & 15 & 7 & 52 \\
\hline Bottom & 1 & 17 & 7 & 1 & 15 & 11 & 52 \\
\hline Total & 17 & 83 & 33 & 11 & 100 & 59 & 303 \\
\hline
\end{tabular}

Table 10

Answers for the expected location in comparison to the experimental conditions.

\begin{tabular}{lrrrrrrr}
\hline \multicolumn{7}{c}{ Expected location } & \multirow{2}{*}{ Total } \\
\cline { 2 - 6 } & Right & Left & Above & Below & Top & Bottom & \\
\cline { 1 - 5 } Experimental & conditions & & & & & & \\
Right & 37 & 3 & 3 & 5 & 1 & 2 & 51 \\
Left & 11 & 17 & 12 & 4 & 5 & 2 & 47 \\
Above & 8 & 4 & 14 & 11 & 2 & 4 & 51 \\
Below & 14 & 1 & 6 & 25 & 5 & 3 & 50 \\
Top & 16 & 3 & 7 & 12 & 14 & 0 & 52 \\
Bottom & 18 & 1 & 9 & 13 & 6 & 5 & 52 \\
Total & 104 & 29 & 51 & 70 & 33 & 16 & 303 \\
\hline
\end{tabular}

\section{References}

Alexa, 2010. Alexa - The Web Information Company. Top Sites by Category: Shopping. <http://www.alexa.com/topsites/category/Top/Shopping>.

Bargas-Avila, J., Hornbæk, K., 2011. Old wine in new bottles or novel challenges? A critical analysis of empirical studies of user experience. In: CHI '11 Proceedings of the 2011 Annual Conference on Human Factors in Computing Systems, pp. 2689-2698.

Bargas-Avila, J., Oberholzer, G., Schmutz, P., de Vito, M., Opwis, K., 2007. Usable error message presentation in the World Wide Web: do not show errors right away. Interacting with Computers 19 (3), 330-341.

Bargas-Avila, J., Brenzikofer, O., Roth, S., Tuch, A., Orsini, S., Opwis, K., 2010. Simple but crucial user interfaces in the world wide web: introducing 20 guidelines for usable web form design. In: Matrai, R. (Ed.), User Interfaces. INTECH, pp. 1-10.

Bargas-Avila, J., Brenzikofer, O., Tuch, A., Roth, S., Opwis, K., 2011a. Working towards usable forms on the world wide web: optimizing date entry input fields. Advances in Human-Computer Interaction (Article ID 202701)

Bargas-Avila, J., Brenzikofer, O., Tuch, A., Roth, S., Opwis, K., 2011b. Working towards usable forms on the world wide web: optimizing multiple selection interface elements. Advances in Human-Computer Interaction, 12 (Article ID 347171).

Bargas-Avila, J., Orsini, S., Piosczyk, H., Urwyler, D., Opwis, K., 2011c. Enhancing online forms: use format specifications for fields with format restrictions to help respondents. Interacting with Computers 23 (1), 33-39.

Becker, S., Mottay, F., 2001. A global perspective on web site usability. IEEE Software $18(1), 54-61$.

Biddle, T., 2007. Usability Tip: Placement of Error Messages. <http:// www.articlestree.com/web-design/usability-tip-placement-of-error-messagestx324629.html>

Brown, P., 1983. Error messages: the neglected area of the man/machine interface. Communications of the ACM 26 (4), 246-249.

Ceaparu, I., Lazar, J., Bessiere, K., Robinson, J., Shneiderman, B., 2004. Determining causes and severity of end-user frustration. International Journal of HumanComputer Interaction 17 (3), 333-356.

Christian, L., Dillman, D., Smyth, J., 2007. Helping respondents get it right the first time: the influence of words, symbols, and graphics in web surveys. Public Opinion Quarterly 71 (1), 113-125.

Couper, M., Tourangeau, R., Conrad, F., Crawford, S., 2004. What they see is what we get: response options for web surveys. Social Science Computer Review 22 (1), 111-127.

Crawford, S., McCabe, S., Pope, D., 2005. Applying web-based survey design standards. In: Technology Applications in Prevention. Routledge, pp. 43-64.

Das, S., McEwan, T., Douglas, D., 2008. Using eye-tracking to evaluate label alignment in online forms. In: Proceedings of the 5th Nordic Conference on Human-Computer Interaction: Building Bridges. ACM New York, NY, USA, pp. 451-454.

Featherstone, D., 2005. Form Error Messages. <http://simplyaccessible.org/article/ form-error-messages>.

Grüner, H., 2008. Configural Frequency Analysis. <http://gruener.userpage.fuberlin.de/spss-dialogs.htm>

Hart, S., Staveland, L., 1988. Development of NASA-TLX (Task Load Index): Results of empirical and theoretical research. In: Human Mental Workload. Amsterdam, Holland, pp. 139-183.

Healey, B., 2007. Drop downs and scroll mice: the effect of response option format and input mechanism employed on data quality in web surveys. Social Science Computer Review 25 (1), 111.

Heerwegh, D., Loosveldt, G., 2002. An evaluation of the effect of response formats on data quality in web surveys. Social Science Computer Review 20 (4), 471.

Hudson, W., 2001. Designing for the grand illusion. ACM SIGCHI Bulletin - A Supplement to Interactions.

ISO-9241, 1996-2002. Ergonomic requirements for office work with visual display terminals (parts 1-17).

Jarrett, C., 2008. How Should I Handle Errors in Online Forms? <http:// www.formsthatwork.com/formerrors>.

Jarrett, C., Gaffney, G., 2008. Forms that Work: Designing Web Forms for Usability. Morgan Kaufmann.

Lang, P.J., 1980. Behavioral treatment and bio-behavioral assessment: computer applications. In: Sidowski, J.B., Johnson, J.H., Williams, T.A. (Eds.), Technology in Mental Health Care Delivery Systems. Ablex Publishing, Norwood, NJ, pp. 119137.

Lazar, J., Huang, Y., 2003, . Improved Error Message Design in Web Browsers, Human Factors and Web Development, second ed. Lawrence Erlbaum Associates, Mahwah, NJ.

Linderman, M., Fried, J., 2004. Defensive Design for the Web: How to Improve Error Messages, Help, Forms, and Other Crisis Points. New Riders Publishing.

Mockovak, W., 2005. Comparing the Effectiveness of Alternative Approaches for Displaying Edit-Error Messages in Web Forms. Tech. Rep.

Nielsen, J., 2001. Error Message Guidelines. <http://www.useit.com/alertbox/ 20010624.html>.

Padilla, M., 2005. Design Interactive Error Handling for Web Apps. <http:// www.ibm.com/developerworks/library/wa-errhand/index.html?ca=drstp0705>.

Pauwels, S., Hübscher, C., Leuthold, S., Bargas-Avila, J., Opwis, K., 2009. Error prevention in online forms: use color instead of asterisks to mark required fields. Interacting with Computers 21 (4), 257-262. 
Penzo, M., 2006. Label Placement in Forms. <http://www.uxmatters.com/MT/ archives/000107.php>

Recabarren, M., Nussbaum, M., 2010. Exploring the feasibility of web form adaptation to users' cultural dimension scores. User Modeling and UserAdapted Interaction 20 (1), 87-108.

Reja, U., Manfreda, K., Hlebec, V., Vehovar, V., 2003. Open-ended vs. close-ended questions in web questionnaires. Advances in Methodology and Statistics 19, 159-177.

Roth, S., Schmutz, P., Pauwels, S., Bargas-Avila, J., Opwis, K., 2010. Mental models for web objects: Where do users expect to find the most frequent objects in online shops, news portals, and company web pages? Interacting with Computers 22 (2), 140-152.

Simons, D., Rensink, R., 2005. Change blindness: Past, present, and future. Trends in Cognitive Sciences 9 (1), 16-20.

StumbleUpon, 2010. Discovery Engine, Category: Shopping. <http:// www.stumbleupon.com/choosetopics/>.

Tuch, A., Roth, S., Hornbaek, K., Opwis, K., Bargas-Avila, J., in press. Is beautiful really usable? Toward understanding the relation between usability, aesthetics, and affect in $\mathrm{HCl}$. Computers in Human Behavior. http://dx.doi.org/10.1016 j.chb.2012.03.024.

Tullis, T., Pons, A., 1997. Designating required vs. optional input fields. In: Conference on Human Factors in Computing Systems. ACM New York, NY USA, pp. 259-260.

Tzeng, J., 2004. Toward a more civilized design: studying the effects of computers that apologize. International Journal of Human-Computer Studies 61 (3), 319345.

Wenger, M., 1991. On the rhetorical contract in human-computer interaction. Computers in Human Behavior 7 (4), 245-262.

Wilska, E., 2004. Non-Fatal Errors: Creating Usable, Effective Error Messages. <http://www.writersua.com/articles/message/index.html>

Wroblewski, L., 2008. Web Form Design: Filling in the Blanks. Rosenfeld Media.

Yom, M., Wilhelm, T., 2004. WOOS-Ein Messinstrument für die wahrgenommene Orientierung in Online-Shops. Mensch \& Computer 43-53. 